\title{
Asymbiotic Germination of Phalaenopsis cv. 'Dublin' Seeds in Relation to Pollination Months and Nutrient Media
}

\author{
Khosro BALILASHAKI ${ }^{1}$, Roohangiz NADERI ${ }^{1}$, Saikat GANTAIT ${ }^{2 *}$, Maryam VAHEDI ${ }^{1}$ \\ ${ }^{1}$ Department of Horticultural Science, Faculty of Agricultural Sciences and Engineering, College of Agriculture and Natural Resources, University of Tehram, Karaj 4111, Iran; \\ khosrobali@alumni.ut.ac.ir; maderi@ut.ac.ir; mary.vahedi@ut.ac.ir \\ ${ }_{2}^{2}$ Sasya Shyamala Krishi Vigyan Kendra, Ramakrishna Mission Vivekananda University, Arapanch, Sonarpur, Kolkata 700150, West Bengal, India; \\ saikatgantait@yahoo.com (*correspondingauthor)
}

\begin{abstract}
Orchids are one of the most popular flowers in the world and Phalaenopsis genus shares maximum sales in global floral industry among them. Successful pollination, followed by capsule formation and in vitro seed germination, is a powerful tool for mass propagation of orchids. In the present study, flowers of Phalaenopsis cv. 'Dublin' were self-pollinated artificially during the months of November, December, January, February, March and April. The influence of pollination month over capsule formation was assessed and it was observed that pollination during December resulted as maximum with $86 \%$ capsule formation, followed by $72 \%$ during January. However, only $40 \%$ successful capsule formation occurred following pollination during April. Seeds from mature capsules developed through artificial pollination in different months were cultured on three different culture media (modified Chen, 1/2Murashige Skoog and Vacin-Went medium) for in vitro germination. A maximum of $97 \%$ germination of seeds (collected from capsules developed through pollination in January) was recorded on modified Chen medium. Nevertheless, the earliest initiation of germination occurred around 12 days of culture when seeds were harvested from capsules (derived through pollination in December) and inoculated on modified Chen medium. The plantlets developed following germination were rooted on $1 / 2 \mathrm{MS}$ supplemented with $2.5 \mathrm{~g} / \mathrm{l}$ activated charcoal. Subsequently, well-rooted plantlets were acclimatized on potting mixture that contained cocopeat, charcoal, industrial cartridge and the bites of yonolit (1:1:2:4; $v / v)$ with a very high rate of success. A seed culture protocol that crops a bulk number of seedlings in a short time span is advantageous and the present report eventually provides such simple yet proficient method that can be further tested.
\end{abstract}

Keywords: acclimatization, capsule, in vitro, modified Chen medium, orchid, rooting, seed culture

\section{Introduction}

Phalaenopsis orchids, owing to large, colorful and adaptable flowers, are highly valued as potted and cut flowers in global flower industry (Batchelor, 1982). Phalaenopsis hybrids are the outcome of intensive breeding of plants having growing ornamental importance. In the commercial arena of horticulture, Phalaenopsis hybrids have ascertained their merit being the most profitable orchid's hybrids with their array of variable colour of inflorescence accompanied by their adaptableness to regular room environment. As reviewed by Lesar et al. (2012), the Royal Horticultural Society, International Cultivar Registration Authority for Orchid Hybrids registered 643 new varieties in 2010, which otherwise means that there are altogether just about 45,000 varieties of Phalaenopsis.

Propagation via seed germination denotes the fundamental adept approach for orchid breeding (Stewart and Kane, 2006). Nevertheless, orchid seeds carry undeveloped endosperm (Prutsch et al., 2000) and they are obligatorily symbiotic with some kinds of fungi, for naturally germination. Orchid seeds can also be germinated well in a culture medium devoid of mycorrhizal fungi. In vitro seed germination of commercially imperative orchid hybrids has been considered as an efficient method for large-scale propagation. In vitro seed germination protocols have been established for numerous orchid species and hybrids since Knudson (1946) first introduced it. Furthermore, asymbiotic germination has been proven to be an ideal system to study physiological effects of photoperiod, temperature, mineral nutrition and medium on germination and subsequent development (Adritti and Ernst, 1993). Even more, in vitro seed germination and seedling developmental stages were reported to be influenced by asymbiotic germination media (Pongener and Deb, 2009); although many of these media have only minor differences in composition, growth and development of species may be significantly affected. Besides photoperiod and basal media, other factor like seeds age, organic carbon concentration, nitrogen level and plant growth regulators have also shown marked effects on seed germination (Deb, 2006).

Few reports are attainable on seed germination of Phalaenopsis (Mweetwa et al., 2008; Balilashaki et al., 2015) 

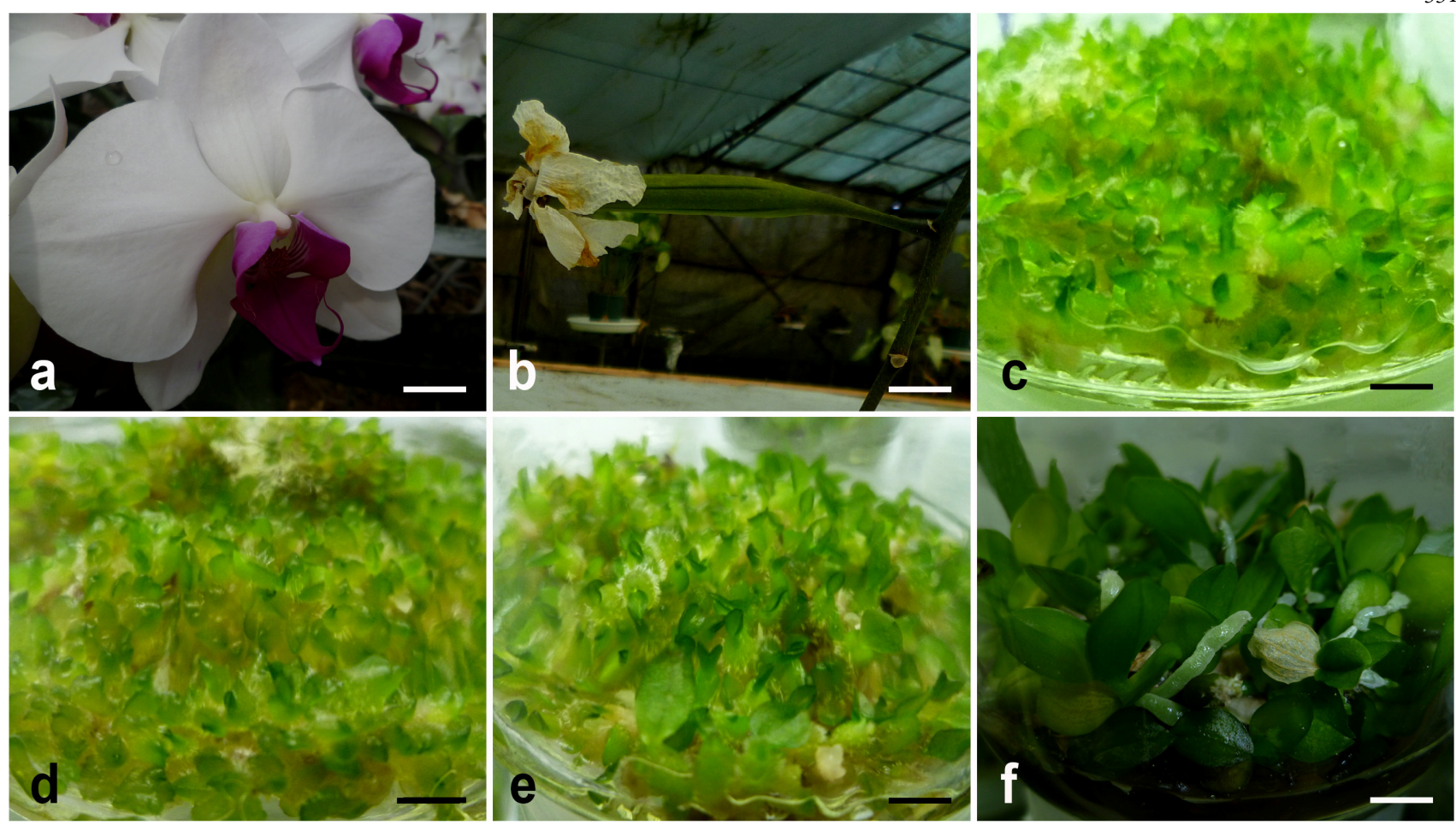

Fig. 1. Pollination, post-pollination capsule formation and asymbiotic seed germination in Phalaenopsis cv. 'Dublin'; a) pollinated flower, b) post-pollination development of capsule, $\mathbf{c}$ ) seed germination on Chen medium, d) seed germination on Vacin-Went medium, e) seed germination on $1 / 2 \mathrm{MS}$ medium, $\mathbf{f}$ ) in vitro rooting of germinated plantlets on $1 / 2 \mathrm{MS}$ with $2.5 \mathrm{~g} / \mathrm{l}$ activated charcoal

and no report ensues on the asymbiotic or symbiotic seed germination requirements of Phalaenopsis 'Dublin', an admired hybrid of Middle East. Considering the importance of asymbiotic seed germination system for mass propagation, the present study aimed at optimizing a reproducible system of in vitro seed germination of Phalaenopsis 'Dublin' and in due course the influence of pollination time and type of medium on capsule formation and seed germination frequency, respectively, were assessed.

\section{Materials and Methods}

Phalaenopsis 'Dublin' (Fig. 1a) plants at their full bloom stage, growing in greenhouse (North of Iran), were used for pollination in the current study. During the onset of the months of November, December, January, February, March and April flowers from one-year-old plants (of circa $20-25 \mathrm{~cm}$ height) were marked and artificially pollinated through removing the anther cap and pollinia by forceps and successively setting the pollinia above the stigma of the same fully opened flower. During every pollination month, 15 flowering plants were selected and 5 flowers from each branch were randomly pollinated. The number of capsule developed during the following month after pollination was recorded in order to measure the effect of pollination time on capsule development.

Undehisced matured capsules were collected after five months from the pollination (completely swollen, dried with dark brown colour) (Fig. 1b). Five capsules from each of the 15 plants were randomly picked and were stored in air-tight plastic packs before they were surface sterilized through washing with $20 \%(v / v)$ commercial bleach (containing 1\% sodium hypochlorite) and one drop of Tween-20 per liter for $10 \mathrm{~min}$, followed by dipping in $70 \%$ ethanol for 30 seconds under aseptic condition.

Seeds were isolated by softly teasing the surface sterilized slitcapsules with scalpel and cultured on different media: the surface of half-strength Murashige and Skoog (1962) (MS), VacinWent (VW) (Vacin and Went, 1949) or modified (supplemented with pepton) Chen medium (Chen et al., 1999), solidified with $0.25 \%(w / v)$ Gelrite (Balilashaki et al., 2015). The media $\mathrm{pH}$ were corrected to 5.7 before autoclaving at $121^{\circ} \mathrm{C}$ and $105 \mathrm{~kg} \mathrm{~cm}^{-2}$ for $15 \mathrm{~min}$. Circa 100 seeds were cultured on the medium of each culture vessel which were maintained at $25 \pm 2$ ${ }^{\circ} \mathrm{C}$ under cool white fluorescent light $\left(50 \mu \mathrm{mol} / \mathrm{m}^{2} / \mathrm{s}\right.$ irradiance) with a 16-h photoperiod. Percent of germinated seeds was documented 60 days following in vitro culture. Initiation of germination was considered when embryos engorged from testa with augmented feature. The germination efficiency of seeds was recorded underneath a light microscope.

Following seed germination and subsequent development, the plantlets were cultured for in vitro rooting on $1 / 2 \mathrm{MS}$ medium supplemented with $2.5 \mathrm{~g} / \mathrm{l}$ activated charcoal. The rooted plantlets were transferred to pots containing sterile mixture of either cocopeat, charcoal, industrial cartridge and the bites of yonolit $(1: 1: 2: 4 ; v / v)$, or cocopeat and charcoal $(5: 1 ; v / v)$ for acclimatization. The pots were enclosed with close-fitting plastic cover to avoid desiccation and opened after two months for postacclimatization transfer into greenhouse.

A completely randomized design with 10 replicates per treatment was followed for the present experiment. Each replicate consisted of five flower samples for the capsule formation experiments, and 100 seeds for the germination experiments. The data for capsule formation, days to germination initiation and germination percentage were 
332

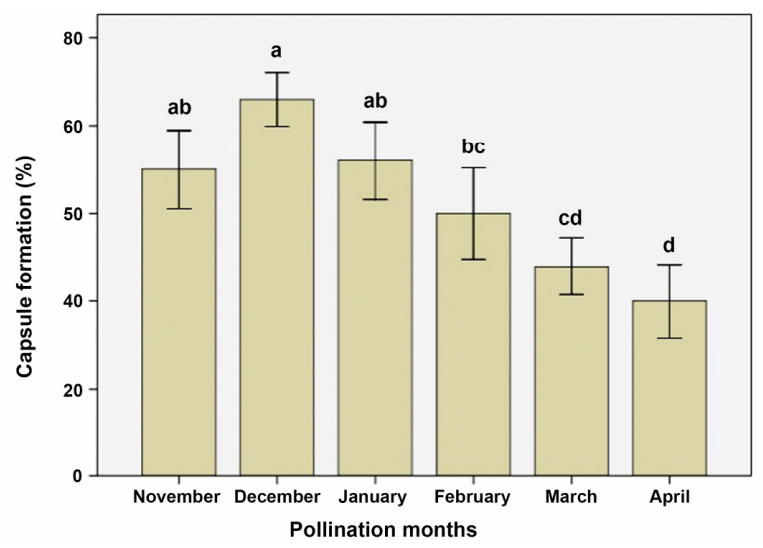

Fig. 2. Influence of pollination time on post-pollination capsules formation frequency (\%)

Data signify (mean \pm standard error bar) 5 flowers per treatment in 10 replicated experiments. Mean column with the same letter are not significant at $\mathrm{P} \leq 0.05$ based on one-way analysis of variance (ANOVA) followed by Tukey's test. Data expressed as percentage were transformed using arcsine prior to ANOVA and converted back to the original scale for demonstration in the histobar (Compton, 1994).

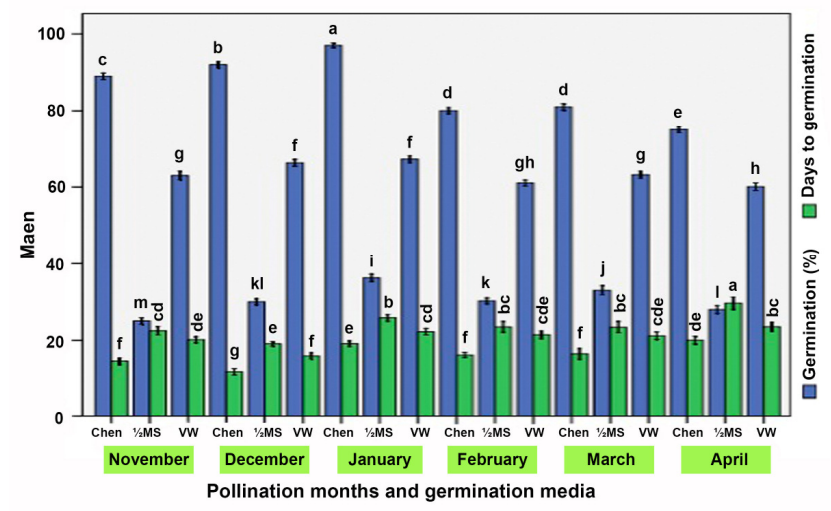

Fig. 3. Influence of pollination time and media on asymbiotic seed germination frequency (\%) and speed of germination Data signify (mean \pm standard error bar) 5 flowers per treatment in 10 replicated experiments. Mean column with the same letter are not significant at $\mathrm{P} \leq 0.05$ based on one-way analysis of variance (ANOVA) followed by Tukey's test. Data expressed as percentage were transformed using arcsine prior to ANOVA and converted back to the original scale for demonstration in the histobar (Compton, 1994).

analyzed through one-way analysis of variance (ANOVA) and the means were compared by the Tukey's test (PC version Origin 7.0 Northampton, MA, USA) at 5\% probability levels using SPSS (Version 11, SPSS Inc. Chicago, USA) software. Percentage of capsule formation and seed germination data were transformed using arcsine prior to ANOVA and converted back to the original scale to normalize variation (Compton, 1994).

\section{Results and Discussion}

The present report ensures the mass propagation of Phalaenopsis cv. 'Dublin' through optimizing a simple yet reproducible protocol for in vitro asymbiotic seed germination and ensuring the maximum capsule formation prior to germination of seeds. Under the current experimental conditions, the earliest sign of success in pollination was petals wilting, which were seen after pollination (Fig. 1b). According to Goh et al. (1985), pollination and emasculation trigger ethylene production by flowers and result in rapid wilting. However, the maximum percentage of capsule formation was achieved following pollination during the onset of January (97\%), followed by December (86\%) (Fig. 2). It was significant to observe that both winter months displayed a similar trend in promoting the success of pollination in terms of post-pollination capsule development frequency. On the contrary, there was a sharp decline in capsule formation with the progress of pollination months following February, and pollination in April resulted in least capsule formation (40\%) (Fig. 2). It is well defined from the present study that the colder months are commonly correlated with higher success of pollination, while warmer months are associated to fewer capsule productions. According to McCall and Primack (1992) environmental circumstances considerably affect the fruit/seed development that was noticeably manifested from the outcomes of the current experiment. Further, the advantageous impact of cooler seasons on efficacious post-pollination capsule development coroborated by the studies of

Vaknin et al. (1996) and Balilashaki et al. (2015), who reported that the frequency of fruit set was lesser in summer in comparison to winter ones in Loranthus acaciae Zucc. (Loranthaceae) and Phalaenopsis respectively.

Seeds from mature capsules were cultured on modifiedChen, Vacin-Went or $1 / 2 \mathrm{MS}$ media. A significant variation was observed among the media both in terms of germination frequency and days to germination. There are previous reports that described that the in vitro germination rate of orchid species was significantly influenced by basal medium used (Islam et al., 2003). The germination frequency might be further promoted by the growth stage of capsules used as in the current study. In accordance to several observations, Schwallier et al. (2011) indicated that the germination rate of seeds taken from young capsules was slower and more variable than that of the mature capsules. The highest frequency of germination percentage (97\%) was achieved on modified-Chen medium (Fig. 1c) supplemented with pepton plus gelrite $(2.5 \mathrm{~g} / \mathrm{l})$ instead of agar, whereas the $1 / 2 \mathrm{MS}$ medium showed the lowest frequency (25.05\%) (Fig. 1e) of seed germination (Fig. 3). The results of the present study correspond with those of Lo et al. (2004) who recommended the usage of coconut water, yeast extract, potato extract and peptone as organic additives for enhanced seed germination and protocorm growth in orchids (Ang and Yong, 2005). The addition of peptone to Chen medium presumably increased seed germination of Phalaenopsis cv. 'Dublin' in comparison to that of the other two media employed. Peptone as an organic nitrogen source can increase seedling development by supplying auxin-like compounds or various amino acids (Kauth et al., 2006). Considering the impact of capsules derived from different pollination months on seed germination it was found that winter months (November-January) are more favorable, associated with higher (89-97\%) and faster seed germination (circa 12-19 days), than the warmer months (February-April) (74-81\%; circa 22-30 days) (Fig. 3). It is noteworthy to mention that the similar trend was observed during post-pollination capsule setting in the study.

Promotional role of activated charcoal during in vitro rooting was similarly evidenced in an earlier study of Gantait et al. (2009) with Vanilla planifolia (Orchidaceae). Regenerated plantlets from seed cultures were rooted with $100 \%$ success in $1 / 2 \mathrm{MS}$ medium fortified with $2.5 \mathrm{~g} / \mathrm{l}$ activated charcoal (Fig. 1f). Subsequently, in the current study a success of $99 \%$ post- 
acclimatization survival was achieved when well rooted plantlets were transferred and grown for two months on the blend of cocopeat, charcoal, industrial cartridge and the bites of yonolit $(1: 1: 2: 4 ; v / v)$, whereas acclimatization on cocopeat and charcoal $(5: 1 ; v / v)$ resulted in $96 \%$ survival.

\section{Conclusions}

Our study demonstrated that apposite time of pollination and favorable in vitro culture media promoted capsule setting and large-scale seed germination of Phalaenopsis cv. 'Dublin'. In a commercial backdrop a seed culture protocol that crops a bulk number of seedlings in a short time span is advantageous and will be profitable to the commercial orchid growers. In that context, the present report eventually provides such simple yet proficient protocol that can be tested at commercial level.

\section{Acknowledgements}

Authors concede the research assistance from the Faculty of Agricultural Sciences and Engineering, College of Agriculture and Natural Resources, University of Tehran.

\section{References}

Arditti J, Ernst R (1993). Micropropagation of orchids. John Wiley and Sons, New York.

Ang SL, Yong JW (2005). A protocol for in vitro germination and sustainable growth of two tropical mistletoes. Plant Cell Tissue and Organ Culture 80:221-228.

Balilashaki K, Gantait S, Naderi R, Vahedi M (2015). Capsule formation and asymbiotic seed germination in some hybrids of Phalaenopsis, influenced by pollination season and capsule maturity. Physiology and Molecular Biology of Plants 21:341-347.

Balilashaki K, Naderi R, Kalantari S, Vahedi M (2014). Efficient in vitro culture protocols for propagating Phalaenopsis 'Cool Breeze'. Plant Tissue Culture and Biotechnology 24:191-203.

Batchelor SR (1982). Beginner's Series-20 Phalaenopsis. American Orchid Society Bulletin 51:12.

Chen JT, Chang C, Chang WC (1999). Direct somatic embryogenesis on leaf explants of Oncidium Gower Ramsey and subsequent plant regeneration. Plant Cell Reports 19:143-149.

Compton ME (1994). Statistical methods suitable for the analysis of plant tissue culture data. Plant Cell Tissue and Organ Culture 37:217-242.

Deb CR (2006). Effect of different factors on non-symbiotic seed germination, formation of protocorm-like bodies and plantlet morphology of Cleisostoma racemiferum (Lindl.) Garay. Indian Journal of Biotechnology 5:223-228.

Gantait S, Mandal N, Bhattacharyya S, Das PK, Nandy S (2009). Mass multiplication of Vanilla planifolia with pure genetic identity confirmed by ISSR. International Journal of Plant Developmental Biology 3:18-23.

Goh CJ, Halevy AH, Engel R, Kofranek AM (1985). Ethylene evolution and sensitivity in cut orchid flowers. Scientia Horticulturae 26:57-67.

Islam MO, Rahman ARMM, Matsui S, Prodhan AUD (2003). Effects of complex organic extracts on callus growth and PLB regeneration through embryogenesis in the Doritaenopsis orchid. Japan Agricultural Research Quarterly 37:229-236.

Kauth PJ, Vendrame WA, Kane ME (2006). In vitro seed culture and seedling development of Calopogon tuberosus. Plant Cell Tissue and Organ Culture 85:91-102.

Knudson L (1946). A nutrient for germination of orchid seeds. American Orchid Society Bulletin 15:214-217.

Lesar H, Ceranič N, Kastelec D, Luthar Z (2012). Asymbiotic seed germination of Phalenopsis Blume orchids after hand pollination. Acta Agriculturae Slovenica 99:5-11.

Lo SF, Nalawade SM, Kuo CL, Chen CL, Tsay HS (2004). Asymbiotic germination of immature seeds, plantlet development and ex vitro establishment of plants of Dendrobium tosaensemakino - A medicinally important orchid. In Vitro Cellular and Developmental Biology-Plant 40:528-535.

McCall CM, Primack RB (1992). Influence of flower characteristics, weather, time and day of season on insect visitation rates in three plant communities. American Journal of Botany 79:434-442.

Mweetwa AM, Welbaum GE, Tay D (2008). Effects of development, temperature, and calcium hypochlorite treatment on in vitro germinability of Phalaenopsis seeds. Scientia Horticulturae 117:257-262.

Murashige T, Skoog F (1962). A revised medium for rapid growth and bioassays with tobacco tissue cultures. Physiologia Plantarum 15:473-497.

Pongener A, Deb CR (2010). Asymbiotic culture of immature embryos, mass multiplication of Cymbidium iridioides D. Don. and the role of different factors. International Journal of Pharma \& Bio Sciences 1:1-15.

Prutsch J, Schardt A, Schill R (2000). Adaptations of an orchid seed to water uptake and -storage. Plant Systematics and Evolution 220:69-75.

Schwallier R, Bhoopalan V, Blackman S (2011). The influence of seed maturation on desiccation tolerance in Phalaenopsisam abilis hybrids. Scientia Horticulturae 128:136-140.

Stewart SL, Kane ME (2006). Asymbiotic seed germination and in vitro seedling development of Habenariam acroceratitis (Orchidaceae), a rare Florida terrestrial orchid. Plant Cell Tissue and Organ Culture 86:147-158.

Vacin EF, Went FW (1949). Some pH changes in nutrient solutions. Botanical Gazette 110:605-613.

Vaknin Y, Tov YY, Eisikowitch D (1996). Flowering seasonality and flower characteristics of Loranthus acaciae Zucc. (Loranthaceae): implications for advertisement and bird-pollination. Sexual Plant Reproduction 9:279-285. 